\title{
Cartas das escolhas sagradas: ferramenta para reflexão sobre cuidado paliativo na Estratégia Saúde da Família
}

\author{
Letters of sacred choices: a tool for reflection on palliative care in the Family Health Strategy
}

Cartas de elecciones sagradas: una herramienta para la reflexión sobre los cuidados paliativos en el Estrategia de salud familiar

\section{Dayse Maria de Vasconcelos Rodrigues ${ }^{1 *}$, Ândrea Cardoso de Souza ${ }^{2}$}

Como citar esse artigo. Rodrigues, DMV; de Souza, AC. Cartas das escolhas sagradas: ferramenta para reflexão sobre cuidado paliativo na Estratégia Saúde da Família. Revista Pró-UniverSUS. 2021 Jan./Jun.; 12 (1): 77-79.

\section{Resumo}

Introdução: o câncer configura-se importante problema de Saúde Pública. Tal fato mostra-se mais complexo quando se refere ao usuário com câncer em cuidado paliativo, devido escassez de instituições e profissionais preparados para cuidar desta clientela. Este estudo tem como objetivos: analisar o processo de cuidar dos pacientes em cuidados paliativos oncológicos e em situação de finitude implementado por profissionais da Estratégia Saúde da Família; conhecer a percepção dos profissionais da Estratégia Saúde da Família sobre cuidado paliativo oncológico e processo de finitude; descrever as ações desenvolvidas por esses profissionais que atribuídas por eles como sendo de cuidado paliativo oncológico. Método: pesquisa exploratória de abordagem qualitativa, que terá como cenário duas unidades de Saúde da Família do município de Niterói. Os participantes serão os profissionais das referidas unidades. Para a coleta de dados será utilizada a entrevista semiestruturada. Resultados esperados: suscitar o debate para ampliar as ações desenvolvidas junto aos usuários em cuidados paliativos oncológicos na Saúde da Família e propor estratégias para a implementação deste cuidado neste ponto de atenção à saúde.

Palavras-chave: Oncologia; Cuidados Paliativos; Atenção Primária à Saúde

\begin{abstract}
Introduction: Cancer is an important problem in Public Health. Such fact becomes more complex when it comes to a person with cancer that is in palliative care, due to a lack of institutions and professionals prepared to handle this specific customer. This study aims to goals to analyze how the process of taking care of patients in palliative care happens when it comes to oncology and finitude situation; to know what the Family Health Strategy professionals understand as oncological palliative care and the finitude process; to describe how Family Health Strategy professionals develop activities which they understand as being part of the palliative care. Materials \& Methods: exploratory research with a qualitative approach. The study will have as setting two units of Family Health Strategy in Niterói's County. The participants will be the professionals of Family Health Strategy. To collect data will be used the a semistructured interview. Expected Results: to raise the debate to expand the actions develop with users in palliative oncology care in Family Health and to propose strategies for the implementation of this point of health care. Keywords: Medical Oncology; Paliative Care; Primary Health Care
\end{abstract}

1 Enfermeira. Mestranda do Mestrado Profissional em Ensino na Saúde/MPES, Universidade Federal Fluminense, Niterói, RJ, Brasil. E-mail: daysevasconcelos@gmail.com ORCID: https://orcid.org/0000-0003-3046-4589

2 Enfermeira. Doutora. Professora do Programa de Mestrado Profissional em Ensino na Saúde/MPES, UFF, RJ, Brasil. E-mail: andriacsouza@gmail.com ORCID: https://orcid. org/0000-0002-6549-8634 


\section{Resumen}

Introducción: El cáncer se presenta como un gran problema de salud pública. El hecho es más complejo cuando se refiere al individuo con cáncer en cuidados paliativos, debido a la escasez de instituciones y profesionales preparados. para tratar con estos pacientes específicos. Este estúdio tiene como objetivos analizar cómo profesionales de Estrategia de Salud Familiar manejan el cuidado paliativo y el proceso de finitud en su vida diária; analizar el proceso de cuidado de los pacientes en cuidados oncológicos paliativos y en situación de finitud. Y conocer lo los profesionales entienden como cuidado paliativo oncológico y proceso de finitude. Método: investigación exploratoria, enfoque cualitativo. Tendrá como escenario dos unidades de Estrategia de Salud Familiar en el municipio de Niterói. Los participantes serán profesionales de los equipos. Será utilizado el entrevistas planeadas para recolectar los datos. Resultados esperados: suscitar el debate para ampliar las acciones desarrolladas com los usuários em cuidados oncológicos paliativos em Salud de la Família Y proponer estratégias para la implementación de este cuidado em este punto de atención de la salud.

Palabras clave: AOncologia Médica; Cuidados Paliativos; Atención Primaria de la Salud.

\section{Introdução}

O câncer se dá por condições multifatoriais, e sua origem está relacionada ao crescimento desordenado de células anormais com potencial invasivo no organismo. Em virtude de sua fisiopatologia possui características desafiadoras e diferenciadas de outras doenças crônicas, devido sua característica em muitos casos, incapacitantes, Além de dores intensas, mutilações e do elevado número de mortes atribuídas ao câncer ${ }^{1}$.

O Instituto Nacional de Câncer ${ }^{2}$, em recente estimativa realizada em 2019, apontou que o Brasil terá 625 mil novos casos de câncer a cada ano do triênio 2020-2022 $2^{2}$.

Distante do que é preconizado pela Política de Humanização e das tecnologias leves de cuidado, o tratamento ainda tem o foco centrado no câncer e não no sujeito com câncer. $\mathrm{O}$ cuidado aos pacientes com câncer deveria ser pensado no contexto territorial o qual o paciente está inserido, contemplar aspectos físicos, emocionais, sociais, espirituais e culturais, no sentido de promover uma atenção integral, entendendo saúde de forma ampliada. É preciso contemplar esses aspectos na atenção aos usuários com câncer visto que essas dimensões da vida são afetadas.

Estar diante dessa doença, da incerteza do tratamento por vezes doloroso e prolongado na maioria dos casos acompanhado de angústia, torna o sujeito frágil, sem perspectiva de futuro e vislumbrando a morte como à única saída, o que tende a piorar o sistema biológico e emocional do paciente. E de certa forma, isso corrobora para a percepção do câncer como sinônimo de morte.

Vida e morte fazem parte da existência humana. E ao adoecer por câncer, o indivíduo confronta-se fortemente com a possibilidade da morte, exacerbando assim, sua fragilidade e vulnerabilidade emocional. Contudo, aceitar e compreender a morte, do ponto de vista físico, seja por causas naturais, idade avançada, por causas externas e ou em decorrência de doenças, no caso deste estudo o câncer, não consiste em uma tarefa fácil ${ }^{3}$.

O sujeito que se encontra em cuidado paliativo oncológico de fato possui sua vida ameaçada pela evolução da sua doença e experencia um processo ativo de finitude e os profissionais precisam estar qualificados para o cuidado destes usuários nos diferentes níveis de complexidade do sistema.

Considerando que o Sistema Único de Saúde (SUS) preconiza que a porta preferencial do sistema seja a Atenção Básica e mais especificamente a Estratégia Saúde da Família (ESF) é preciso que às equipes estejam preparadas para identificar usuários com queixas e sintomatologias sugestivas de neoplasias, ofertarem o cuidado adequado e sendo capaz de produzir ações efetivas que por vezes, extrapolam este âmbito de atenção. Entretanto é nesse ponto que as primeiras queixas são investigadas e inicia-se a investigação diagnóstica, sendo necessário o referenciamento para unidades de saúde de média e alta complexidade no âmbito do SUS.

Porém, é preciso considerar que este usuário está sob a responsabilidade sanitária de uma unidade de ESF e que esta deverá se encarregar da atenção à saúde de modo longitudinal, contemplando um cuidado integral. Apesar das diretrizes da Política Nacional de Atenção Básica $^{4}$, é sabido que o atendimento de qualidade por vezes é negligenciado.

Um grande desafio da atualidade consiste em produzir um cuidado eficaz aos usuários com câncer, clientela que tende continuar aumentando, já que o câncer é considerado um problema de Saúde Pública. E este se torna ainda maior diante dos usuários com câncer avançado, sem possibilidade de tratamento terapêutico curativo e que necessitam de cuidado paliativo ou já se encontram em processo de finitude.

A Educação Permanente em Saúde (EPS) é uma ferramenta com potencialidade para proporcionar 
trocas de experiências e de problemas vivenciados, proporcionar aprendizados nas unidades de saúde que oportunizem o enfrentamento do cotidiano por meio de uma atuação crítica, reflexiva, propositiva, compromissada e tecnicamente competente, no cuidado prestado ao usuário em cuidado paliativo oncológico ${ }^{5}$.

O objeto desse estudo consiste conhecer como é o processo de cuidado paliativo oncológico junto ao usuário em processo de finitude nas unidades da ESF.

Objetivo Geral: Analisar o processo de cuidar dos pacientes em cuidados paliativos oncológicos e em situação de finitude adotado por profissionais da Estratégia Saúde da Família.

Objetivos Específicos: 1- Conhecer a percepção dos profissionais da Estratégia Saúde da Família sobre cuidado paliativo oncológico e processo de finitude; 2Descrever as ações desenvolvidas pelos profissionais da ESF, as quais os mesmos atribuem como sendo de cuidado paliativo oncológico.

\section{Metodologia}

Oestudo se caracteriza como pesquisa exploratória de abordagem qualitativa ${ }^{6}$. Terá como cenário duas unidades de ESF situadas no município de Niterói.

Os participantes serão profissionais da ESF que atuam na atenção aos usuários em cuidado paliativo na ESF. Este estudo atende as recomendações da Resolução No 466, de 12 de dezembro de 2012, do Conselho Nacional de Saúde ${ }^{7}$. Com aprovação do CEP/ UFF número 4.520.766.

A coleta de dados será feita por meio de análise de entrevistas semiestruturadas 8 .

A análise dos dados será guiada pelo referencial da análise temática. Com a análise das entrevistas intenciona-se construir um mapa analítico que permita identificar os principais nós críticos para um cuidado integral e qualificado aos pacientes em cuidado paliativo oncológico e em situação de finitude na ESF

\section{Resultados Esperados}

Espera-se que com a realização do estudo seja possível ampliar os debates sobre esta temática e chamar atenção dos gestores e Instituições de Ensino Superior para a necessidade de qualificar a formação no sentido de inserir esse conteúdo nos cursos. Além de contribuir para a incorporação de práticas de cuidado aos usuários em cuidados paliativos oncológicos e em processo de finitude na Saúde da Família.

\section{Referências}

1. Kersul, A.P. Enfrentamento do câncer: riscos e agravos. Belo Horizonte: NESCON UFMG.p12 2014.Disponível em: https://www.nescon. medicina.ufmg.br/biblioteca/imagem/enfretamento-cancer-riscos-agravos. pdf . Acessado em :20/01/2020

2. INCA, Instituto Nacional De Câncer José Alencar Gomes Da Silva. Estimativa 2019: incidência de câncer no Brasil. Coordenação de Prevenção e Vigilância - Rio de Janeiro: INCA, 2019.

3. Lima L.M, Bielemann V.L.M, Schwartz E, Viegas A.C, Santos B.P. Adoecer de câncer: o agir e o sentir do grupo familiar. Ciênc Cuid Saúde. 2012, p.106-12. Disponível em: http://www.revenf.bvs.br/pdf/ccs/v11n1/14. pdf.

4. Brasil. Ministério Da Saúde. Secretaria de Atenção à Saúde Departamento de Atenção Básica. Política Nacional de Atenção Básica. Brasília. 2012. Disponível: https://bvsms.saude.gov.br/bvs/publicacoes/ politica_nacional_atencao_basica.pdf . acessado em: 20/01/2020.

5. Ceccim R.B. Educação permanente em saúde: desafio ambicioso e necessário. Interface - Comunic, Saúde, Educ 9(16): 2005, p.161-168.

6. 2008 .

Gil A.C. Como elaborar projetos de pesquisa. São Paulo: Atlas;

7. CNS, Conselho Nacional de Saúde. Resolução 466 DO CNS. Disponível em: https://conselho.saude.gov.br/ultimas_noticias/2013/06 jun_14_publicada_resolucao.html. Acesso em: 25/01/2020.

8. Marconi, M. A.; Lakatos, E.M. Fundamentos de metodologia científica. 7 ed. São Paulo: Atlas; 2010. 